\title{
The role of corifollitropin alfa in controlled ovarian stimulation for IVF in combination with $\mathrm{GnRH}$ antagonist
}

This article was published in the following Dove Press journal:

International Journal of Women's Health

5 August 201I

Number of times this article has been viewed

\author{
Ayse Seyhan \\ Baris Ata \\ Division of Reproductive \\ Endocrinology and Infertility, \\ Department of Obstetrics and \\ Gynecology, McGill University, \\ Montreal, QC, Canada
}

Correspondence: Baris Ata MUHC Reproductive Centre, Royal Victoria Hospital 687 Pine Avenue West, Women's Pavilion Room F6.58 H3A

IAI Montreal, QC, Canada

$\mathrm{Tel}+|5| 48431650$

$\mathrm{Fax}+|5| 4843$ |496

Email baris.ata@muhc.mcgill.ca
Abstract: Corifollitropin alfa is a synthetic recombinant follicle-stimulating hormone (rFSH) molecule containing a hybrid beta subunit, which provides a plasma half-life of $\sim 65$ hours while maintaining its pharmocodynamic activity. A single injection of corifollitropin alfa can replace daily FSH injections for the first week of ovarian stimulation for in vitro fertilization. Stimulation can be continued with daily FSH injections if the need arises. To date, more than 2500 anticipated normoresponder women have participated in clinical trials with corifollitropin alfa. It is noteworthy that one-third of women did not require additional gonadotropin injections and reached human chorionic gonadotropin criterion on day 8 . The optimal corifollitropin dose has been calculated to be $100 \mu \mathrm{g}$ for women with a body weight $\leq 60 \mathrm{~kg}$ and $150 \mu \mathrm{g}$ for women with a body weight $>60 \mathrm{~kg}$, respectively. Combination of corifollitropin with daily gonadotropin-releasing hormone antagonist injections starting on stimulation day 5 seems to yield similar or significantly higher numbers of oocytes and good quality embryos, as well as similar ongoing pregnancy rates compared with women stimulated with daily rFSH injections. Stimulation characteristics, embryology, and clinical outcomes seem consistent with repeated corifollitropin-stimulated assisted reproductive technologies cycles. Multiple pregnancy or ovarian hyperstimulation syndrome rates with corifollitropin were not increased over daily FSH regimen. The corifollitropin alfa molecule does not seem to be immunogenic and does not induce neutralizing antibody formation. Drug hypersensitivity and injection-site reactions are not increased. Incidence and nature of adverse events and serious adverse events are similar to daily FSH injections. Current trials do not provide information regarding use of corifollitropin alfa in anticipated hyper- and poor responders to gonadotropin stimulation. Although corifollitropin alfa is unlikely to be teratogenic, at the moment data on congenital malformations is missing. Keywords: follicle-stimulating hormone, long acting, controlled ovarian hyperstimulation, in vitro fertilization, assisted reproduction

\section{Introduction}

Infertility affects $10 \%-15 \%$ couples trying to conceive. ${ }^{1,2}$ Assisted reproductive technologies (ART) provide the highest chance of success for those couples. Although the first ART pregnancy was achieved in a natural cycle, it was soon realized that the chances of achieving a pregnancy was related to the number of oocytes available for in vitro fertilization (IVF). ${ }^{3}$ Ovarian stimulation with exogenous gonadotropins quickly became standard practice. The introduction of gonadotropin-releasing hormone $(\mathrm{GnRH})$ agonists (GnRHa) enabled prevention of premature luteinizing hormone (LH) surges observed in stimulated cycles and scheduling oocyte retrieval. Thus, controlled ovarian hyperstimulation (COS) with its two components, gonadotropin and GnRHa, became an integral part of the ART cycle. ${ }^{4}$ COS proved to increase the success rate of IVF, 
and the results achieved by most treatment centers exceed spontaneous conception rates in healthy fertile couples. ${ }^{5}$

The long GnRHa protocol has been the most commonly used COS protocol for about two decades. While effectively preventing premature LH surges and maintaining multifollicular growth, the long GnRHa protocol requires approximately 3 weeks of self injections on the part of the patient. Daily GnRHa injections are started from the midluteal phase of the preceding cycle, and daily gonadotropin injections are added within the first week of menstruation. Both injections are continued until follicles reach adequate size. While GnRHa injections last around 20 days, gonadotropin injections last 10-12 days on average, depending on the individual ovarian response.

Daily injections for 3 weeks are inconvenient and possibly contribute to the stress perceived by the patients. Indeed, infertile patients experience a high level of distress, and their level of anxiety and depression is equivalent to that experienced by women with cancer or heart disease. ${ }^{6}$ Almost one-third of the couples end treatment prematurely because of its psychological burden. ${ }^{7}$ A high proportion of couples drop out even when treatment costs are fully covered by third parties. Importantly, when a mild ART strategy was employed, the association between the baseline anxiety score and drop-out was reduced by $>50 \% .{ }^{8}$ Obviously, simpler and more convenient treatment strategies improve the overall patient experience. Fewer injections may also lessen the complexity and psychological burden of IVF.

The first improvement to shorten treatment duration and decrease the number of injections was the introduction of GnRH antagonists (GnRHant) into ART practice. Different from downregulation induced by GnRHa, GnRHant competitively inhibits GnRH activity at the receptor level and provides rapid prevention of premature LH surges in COS cycles. This enables starting GnRHant injections during the actual COS cycle when premature LH surges are imminent. GnRHant is started either on day 6 of gonadotropin stimulation or when the leading follicle reaches $14 \mathrm{~mm}$, depending on the choice of protocol, and continued until follicular maturity. Compared to 3 weeks of GnRHa injections in the long protocol, most women only need about 5 days on GnRHant injections. The use of GnRHant decreases GnRH analog injections by about 2 weeks. The initial concern about decreased pregnancy rates with GnRHant has subsided, and GnRHant have become the pituitary suppression agent of choice in many ART centers worldwide. ${ }^{9,10}$ In fact, more recent patient-friendly, mild-stimulation protocols all utilize GnRHant to prevent premature LH surges.
The second development which can further simplify ART treatment is the introduction of corifollitropin alfa, a long-acting recombinant follicle-stimulating hormone (rFSH) molecule. A single injection of corifollitropin alfa can replace daily gonadotropin injections for 7 days and can further decrease the number of injections required in a COS cycle. ${ }^{11-15}$

In the remainder of this manuscript a brief review of the pharmacologic properties of corifollitropin alfa will be followed by treatment outcomes of corrifollitropin alfa-stimulated ART cycles. The authors of this paper performed a systematic literature search in the Cochrane Central Register of Controlled Trials, MEDLINE, EMBASE, and OVID MEDLINE ${ }^{\circledR}$ In-Process and Other Non-Indexed Citations databases using combinations of the keywords corifollitropin alfa, follicle stimulating hormone, follicle stimulant, sustained, carboxy terminal peptide, Elonva, ovarian stimulation, controlled, and IVF. We searched the abstracts of the American Society of Reproductive Medicine and the European Society of Human Reproduction and Embryology annual meetings through websites of 'Fertility and Sterility' and 'Human Reproduction', respectively. Reference lists of identified reports were manually searched for other relevant publications.

\section{Corifollitropin alfa - the molecule}

FSH belongs to the glycoprotein hormone family. Other members are LH, human chorionic gonadotropin (hCG), and thyroid-stimulating hormone. Glycoprotein hormones are dimeric proteins consisting of two nonidentical, noncovalently linked alpha and beta subunits. The alpha subunit is common to all family members, whereas the beta subunit is unique to each hormone and confers biologic specificity. ${ }^{16,17}$ With the exception of hCG, the human glycoprotein hormones have a relatively short terminal half-life $\left(t_{1 / 2}\right)$ in vivo. ${ }^{16,18}$ While terminal $\mathrm{t}_{1 / 2}$ of LH and FSH are about 60 minutes and 3-4 hours, respectively, hCG has a terminal $\mathrm{t}_{1 / 2}$ of 25-33 hours. Plasma $\mathrm{t}_{1 / 2}$ of hCG is nearly 10 -fold longer than that of LH. ${ }^{19,20}$ The major structural difference between the two molecules is the 31 amino acids long C-terminal peptide (CTP) that exists in the beta subunit of hCG but not in LH. The hCG-CTP includes four additional O-linked carbohydrate side chains, each of which has two terminal sialic acid residues. ${ }^{21}$ It is the presence of these side chains at the CTP site that confers the longest circulating $t_{1 / 2}$ among glycoprotein hormones to hCG. ${ }^{22,23}$ HCG lacking the CTP sequence has three-fold less bioactivity than native hCG. ${ }^{22}$ Glycosylation of the CTP increases the plasma $t_{1 / 2}$ of $h C G$ by reducing glomerular filtration within the kidney. ${ }^{16,23-25}$ 
The relatively short $t_{1 / 2}$ of the wild-type FSH molecule (WT-FSH) requires once daily dosing for COS. Several different approaches have been used to develop an FSH preparation with a longer plasma $t_{1 / 2}$. Initially it has been suggested that increasing the molecular weight and glycosylation of the WT-FSH could generate more negatively charged forms and decrease glomerular filtration of FSH. Thus, the circulating $t_{1 / 2}$ of the hormone could be prolonged secondary to a decrease in renal clearance. Indeed, increasing the number of glycosylation sites on the FSH molecule with either Nor O-linked moieties extended the plasma $t_{1 / 2}$ as much as two-fold compared with human $\mathrm{rFSH} .{ }^{26,27}$ However, after a certain point it was not possible to further extend plasma $t_{1 / 2}$ by glycosylation. ${ }^{27}$

Boime and colleagues took a novel approach and fused copies of CTP of hCG beta-subunit to the FSH betasubunit. ${ }^{28,29}$ They constructed chimeric genes containing the sequence encoding the CTP of hCG beta subunit fused to the sequence of the human FSH beta subunit. The new FSH beta-CTP sequence was transfected into Chinese hamster ovary $(\mathrm{CHO})$ cells together with the wild-type alpha subunit sequence. Compared to the WT-FSH sequence, the addition of CTP sequences did not significantly affect assembly, secretion, or stimulation of steroidogenesis in vitro. However, the in vivo potency of the chimera-containing CTP (FSH-CTP, corifollitropin alfa) was substantially increased. ${ }^{28}$ Corifollitropin alfa was found to be 10 -fold more biopotent than WT-FSH. ${ }^{29}$ One study demonstrated that a single injection of 10 IU corifollitropin alfa was sufficient to stimulate follicle maturation in rats, such that ovulation could be induced by hCG 52 hours later. In contrast, a single injection of $10 \mathrm{IU}$ WT-FSH was ineffective in increasing ovulatory potential, whereas the same total dose of WT-FSH, administered as four separate 2.5 IU injections 12 hours apart, was as effective as corifollitropin alfa. ${ }^{29}$ These results indicate that maintenance of serum FSH level above the threshold value required to stimulate follicular growth is more important than total dose administered. ${ }^{29}$

Importantly, corifollitropin alfa was found to interact only with the FSH-receptor and lacked any LH activity. ${ }^{26,29,30}$

\section{Pharmacokinetics}

Animal studies have shown that corifollitropin alfa has a comparable pharmacological activity and a prolonged plasma $t_{1 / 2}$ compared with rFSH. ${ }^{28,29}$ Because of its long $t_{1 / 2}$, standardization of corifollitropin alfa activity by means of the classical Steelman-Pohley bioassay does not provide a reliable estimate of its in vivo bioactivity from a pharmacological or clinical perspective. ${ }^{12,31}$ Therefore, pure recombinant corifollitropin alfa dosages are expressed in mass (micrograms) instead of international units. ${ }^{12}$

\section{Phase I studies}

The first human study was conducted in men with hypogonadotropic hypogonadism. ${ }^{32}$ The plasma $t_{1 / 2}$ of corifollitropin alfa in humans was found to be $94.7 \pm 26.2$ hours, approximately two- to three-fold longer than the $\mathrm{t}_{1 / 2}$ of $\mathrm{rFSH} .{ }^{32}$ The plasma $t_{1 / 2}$ of $\mathrm{rFSH}$ has been shown to be $\sim 34 \pm 5$ hours in pituitarysuppressed females. ${ }^{33}$ Compared with $\mathrm{rFSH}$, corifollitropin alfa absorption appeared to be slow, with a time to maximal serum concentration $\left(\mathrm{t}_{\max }\right)$ of $46 \pm 18$ hours after injection. RFSH reaches maximal serum concentrations by $13 \pm 6$ hours after a subcutaneous injection in women. ${ }^{33}$

The first corifollitropin alfa study in women involved 24 healthy women who underwent pituitary suppression with a combined oral contraceptive pill (OCP).${ }^{11}$ Participants were given a single dose of $15,30,60$, or $120 \mu \mathrm{g}$ of corifollitropin alfa on the 21 st day of OCP. The median $t_{\text {max }}$ was 36 hours in the 15,60 , and $120 \mu \mathrm{g}$ groups, and 48 hours in the $30 \mu \mathrm{g}$ group. Plasma $t_{1 / 2}$ ranged between 60 hours in the $30 \mu \mathrm{g}$ group and 75 hours in the $120 \mu \mathrm{g}$ group. After normalizing for the dosage administered, both $t_{\max }$ and $t_{1 / 2}$ were found to be dose independent within the dose range tested. ${ }^{11}$ Earlier studies evaluating pharmacokinetics of $\mathrm{rFSH}$ in healthy women who had pituitary suppression with the same OCP reported that $\mathrm{t}_{\max }$ was 17 hours after a single $300 \mathrm{IU}$ dose of $\mathrm{rFSH},{ }^{34}$ and varied between 7.5 and 8.5 hours following daily injections of $\mathrm{rFSH}$ at doses of 75,150 , or $225 \mathrm{IU}$ for 7 days. ${ }^{35} \mathrm{~T}_{1 / 2}$ of rFSH was reported to be 34.8-36.2 hours in the same study. ${ }^{35}$ In another study, $t_{1 / 2}$ of $\mathrm{rFSH}$ was 37 hours following a single 150 IU injection and 24 hours after 7 days of daily $150 \mathrm{IU}$ injections in pituitary-suppressed women. ${ }^{36}$

Based on the abovementioned results, absorption of corifollitropin alfa appears to be slower than that of $\mathrm{rFSH}$. The reason for slower absorption can be the larger size of the corifollitropin alfa molecule than the rFSH molecule. Regardless, plasma $t_{1 / 2}$ for corifollitropin alfa is consistently longer, reaching twice $t_{1 / 2}$ of rFSH in healthy women with pituitary suppression.

\section{Phase II studies}

In a double-blind, placebo-controlled, randomized trial, 55 oligo-amenorrheic women with World Health Organization Group II anovulatory infertility were randomized to receive either a single injection of $7.5,15,30$, or $60 \mu \mathrm{g}$ corifollitropin alfa or placebo on day $2-3$ of a spontaneous 
or progestagen-induced menstrual bleeding. ${ }^{37}$ While $\mathrm{t}_{\max }$ was consistently around 26.0-28.6 hours in all groups, the mean plasma $t_{1 / 2}$ ranged between 77 and 86 hours in the 15, 30, and $60 \mu \mathrm{g}$ groups, but it was 110 hours in the $7.5 \mu \mathrm{g}$ group. The dose-normalized measures of individual exposure to corifollitropin alfa, calculated with maximal serum concentration $\left(\mathrm{C}_{\max }\right)$ and the area under the curve (AUC) after taking into account individual total serum clearance and volume of distribution were similar for the 15,30 , and $60 \mu \mathrm{g}$ doses. These findings show that the serum concentration of corifollitropin alfa is proportional to the dose within the 15-60 $\mu \mathrm{g}$ dose range. The relatively longer plasma $t_{1 / 2}$ of the corifollitropin alfa after a $7.5 \mu \mathrm{g}$ dose observed in this study was due to lower serum clearance observed in this group. The investigators suggested that this was a "chance finding" and plasma $t_{1 / 2}$ was independent from the dose given. ${ }^{37}$ These results shows that pharmacokinetics of corifollitropin alfa is similar in anovulatory women and healthy women with pituitary suppression, and it is not affected by the endocrine status of recipient.

To date, corifollitropin alfa, in doses ranging 60-240 $\mu \mathrm{g}$, has been given to some 2000 women undergoing IVF treatment in the context of randomized controlled trials (RCT). ${ }^{12-15,38}$ All these studies have used GnRHant to prevent premature LH surges. The pharmokinetic parameters of corifollitropin alfa in IVF patients seem to be consistent with the previous studies, with $\mathrm{C}_{\text {max }}$ being reached on day 2 after injection ( $\mathrm{t}_{\max } 25-46$ hours) and plasma $t_{1 / 2}$ being approximately 65 hours. ${ }^{11,32,37}$ The dose-normalized AUC and dosenormalized $\mathrm{C}_{\max }$ values were similar across the dose range used in these trials. These trials also demonstrated $\mathrm{C}_{\max }$ and AUC values for corifollitropin alfa were dose-proportional for doses ranging from 60 to $240 \mu \mathrm{g} .{ }^{13,38,39}$

Corifollitropin alfa exposure showed an inverse relationship with bodyweight, which was found to be a significant covariate of clearance and volume of distribution. ${ }^{13} \mathrm{C}_{\max }$ and
AUC were calculated to be almost twice as high in a woman with $50 \mathrm{~kg}$ bodyweight (5.12 ng/mL and $735 \mathrm{ng} \cdot \mathrm{h} / \mathrm{mL}$ ) compared with a woman who has a bodyweight of $80 \mathrm{~kg}(2.67 \mathrm{ng} /$ $\mathrm{mL}$ and $402 \mathrm{ng} \cdot \mathrm{h} / \mathrm{mL}){ }^{13}$

Pharmacokinetic characteristics of corifollitropin are summarized in Table 1.

\section{Clinical trials of corifollitropin in ART cycles}

The first live birth following the use of corifollitropin alfa for COS in an ART cycle was reported in a woman who had primary infertility for 7 years. ${ }^{40}$ She received a single $180 \mu \mathrm{g}$ dose of corifollitropin alfa on cycle day 3, which was followed by daily rFSH injections from day 10 of stimulation until hCG administration. GnRHant was used for pituitary suppression. Twelve oocytes were retrieved, 10 were fertilized, and two embryos were transferred, and the patient had a singleton live birth at term. The use of corifollitropin alfa in COS-IVF cycles has been evaluated in four RCTs. ${ }^{12-15}$ The efficacy and safety of repeated COS cycles with corifollitropin was evaluated in a prospective cohort study. ${ }^{41}$

All four RCTs have recruited women with regular menstrual periods and younger than 40 years of age. Women who were underweight or obese according to body mass index, ${ }^{12-15}$ had polycystic ovarian syndrome (PCOS), ${ }^{12-15}$ had high antral follicle count $(>20),{ }^{14,15}$ had elevated follicular phase serum FSH level, ${ }^{14,15}$ had a history of over or poor response to gonadotropin stimulation, ${ }^{13-15}$ had recurrent miscarriage or recurrent implantation failure, ${ }^{13-15}$ had oopherectomy, ${ }^{13-15}$ or smoked $>5$ cigarettes/day were excluded. ${ }^{14,15}$

Pituitary suppression was achieved with GnRHant in all trials, albeit starting day of GnRHant varied in one trial. When women allocated to corifollitropin alfa needed further stimulation to reach hCG criterion, they received daily $\mathrm{rFSH}$ injections from stimulation day 8 on, with dosage depending on the individual trial protocol. HCG administration criterion

Table I Pharmacokinetic characteristics of recombinant FSH preparations

\begin{tabular}{|c|c|c|c|c|}
\hline Population & FSH preparation & Dose & $\mathbf{t}_{\max }$ (hours) & $\mathbf{t}_{1 / 2}$ (hours) \\
\hline Healthy women with pituitary suppression" & Corifollitropin alfa & $120 \mu \mathrm{g}$ & 36.0 & 74.5 \\
\hline Women with WHO class II anovulation ${ }^{37}$ & Corifollitropin alfa & $60 \mu \mathrm{g}$ & 26.5 & 83.9 \\
\hline Normo-ovulatory women undergoing $\mathrm{IVF}^{13}$ & Corifollitropin alfa & $120 \mu \mathrm{g}$ & 41.2 & 65.3 \\
\hline Normo-ovulatory women $<60 \mathrm{~kg}$ undergoing IVF 15 & Corifollitropin alfa & $100 \mu \mathrm{g}$ & 46.2 & 73.1 \\
\hline Normo-ovulatory women undergoing IVF' ${ }^{14}$ & Corifollitropin alfa & $150 \mu \mathrm{g}$ & 43.8 & 68.2 \\
\hline Gonadotropin deficient women ${ }^{34}$ & Recombinant FSH & $300 \mathrm{IU}$ & 27.0 & 44.0 \\
\hline Gonadotropin deficient women ${ }^{34}$ & Urinary FSH & $300 \mathrm{IU}$ & 21.0 & 38.0 \\
\hline Healthy women with pituitary suppression ${ }^{34}$ & Recombinant $\mathrm{FSH}^{\mathrm{a}}$ & I50 IU & 10.6 & 30.6 \\
\hline
\end{tabular}

Notes: aDaily administration for 7 days. All values after a single dose administration unless otherwise stated.

Abbreviations: FSH, follicle-stimulating hormone; IVF, in-vitro fertilization; $\mathrm{t}_{\max }$, time to reach maximal serum concentration; $\mathrm{t}_{1 / 2}$, plasma half life; WHO, World Health Organization. 
was uniform across the trials, ie, three or more follicles $\geq 17 \mathrm{~mm}$.

Number and size of ovarian follicles were assessed with serial ultrasound examinations. Blood samples were drawn for assessment of serum FSH, LH, estradiol (E2), inhibin B, and progesterone levels during the stimulation period as well as during the luteal phase in one trial. Relevant characteristics of the four RCTs are described in Table 2.

A brief description of individual trials will be followed by the results for different outcome measures.

\section{The feasibility study (FS) ${ }^{12}$}

The first RCT was a Phase II FS evaluating the efficacy and safety of corifollitropin alfa in COS-IVF. ${ }^{12}$ A total of 98 women were randomized to receive a $120(\mathrm{n}=25), 180$ $(\mathrm{n}=24)$, or $240 \mu \mathrm{g}(\mathrm{n}=25)$ injection of corifollitropin alfa on cycle day 2 or 3 . Women who did not reach hCG criterion on day 8 received $150 \mathrm{IU} /$ day $\mathrm{rFSH}$ injections from stimulation day 8 onwards. Women allocated to the control group $(\mathrm{n}=24)$ received $150 \mathrm{IU} /$ day $\mathrm{rFSH}$ injections from the start. Daily GnRHant injections were started on the day when the leading follicle reached $\geq 14 \mathrm{~mm}$ size. Up to three embryos were transferred on day 3 or 5 after fertilization.

\section{The dose-finding study (DFS) ${ }^{13}$}

The second RCT was a DFS evaluating three different doses of corifollitropin alfa. ${ }^{13}$ The investigators attributed the failure to demonstrate a strong dose response effect in the $\mathrm{FS}^{12}$ to the high dose range tested and therefore decreased corifollitropin alfa dose to 60,120 , and $180 \mu \mathrm{g}$ for the DFS. A total of 315 women were randomized and received a single injection of $60 \mu \mathrm{g}$ corifollitropin alfa $(\mathrm{n}=78)$, $120 \mu \mathrm{g}$ corifollitropin alfa $(\mathrm{n}=77), 180 \mu \mathrm{g}$ corifollitropin alfa $(n=79)$, or daily injections of $150 \mathrm{IU}$ rFSH $(n=81)$ from cycle day $2-3$. If women allocated to corifollitropin alfa needed further stimulation to meet the hCG criterion, they received a fixed dose of $150 \mathrm{IU} /$ day $\mathrm{rFSH}$ from stimulation day 8 onwards. Daily GnRHant injections were started on stimulation day 5 due to a relatively high incidence of premature LH surges observed with the flexible GnRHant protocol used in the FS. ${ }^{12}$ The incidence of premature LH surges will be mentioned further on in the Hormonal Profiles section. Up to three embryos were transferred 3-5 days after fertilization.

\section{The ENGAGE trial $^{14}$}

The third RCT was the ENGAGE trial, a multicenter, randomized, double-blind, double-dummy, noninferiority clinical trial investigating whether ongoing pregnancy rate (defined as $>12$ weeks pregnancy with fetal heart beat) achieved with a corifollitropin alfa regimen was within $8 \%$ noninferiority margin compared with a standard daily rFSH regimen. ${ }^{14}$ While the $\mathrm{FS}^{12}$ and the DFS ${ }^{13}$ were mainly Phase II trials which had a focus on the number of oocytes collected, ENGAGE was the first Phase III trial aimed to test ongoing pregnancy rates against a daily $\mathrm{rFSH}$ regimen. Based on the results obtained in the previous trials, the lowest corifollitropin alfa dose necessary to obtain an optimal treatment outcome was calculated to be $100 \mu \mathrm{g}$ for women weighing $\leq 60 \mathrm{~kg}$ and $150 \mu \mathrm{g}$ for women weighing $>60 \mathrm{~kg} .{ }^{42}$ The ENGAGE trial recruited women weighing $>60 \mathrm{~kg}$ up to and including $90 \mathrm{~kg}$. Women received either $150 \mu \mathrm{g}$ corifollitropin alfa $(\mathrm{n}=756)$ or started 200 IU/day rFSH injections $(n=750)$ on cycle day $2-3$. Notably, ENGAGE was the largest double-blind RCT ever conducted in the field of ART. Dose reduction was allowed from day 5 of stimulation for women who were deemed over-responding to stimulation by the local investigators, but due to the double-dummy design, women in the corifollitropin alfa group would actually have placebo dose reduced between days 5-8. GnRHant injections were started on stimulation day 5. The maximum rFSH dose allowed was $200 \mathrm{IU} /$ day for women who needed stimulation after day 7. Coasting was allowed up to 3 days, but the hCG injection had to be withheld for women who had $>30$ follicles of $\geq 11 \mathrm{~mm}$ in any case. Up to two embryos were transferred 3-5 days after fertilization.

\section{The ENSURE trial ${ }^{15}$}

The fourth study was the ENSURE trial, ${ }^{15}$ which had the same design features as the ENGAGE trial, ie, doubleblind, double-dummy, placebo-controlled RCT with the same inclusion and exclusion criteria. Different than the ENGAGE, the ENSURE trial recruited women who weighed $\leq 60 \mathrm{~kg}$. According to the previously mentioned calculations, 268 women allocated to the corifollitropin alfa group received a $100 \mu \mathrm{g}$ injection, while 128 women allocated to the rFSH group started $150 \mathrm{IU} /$ day injections on cycle days 2-3. Dose adjustments were allowed from stimulation day 6 on. The maximum allowed daily $\mathrm{rFSH}$ dosage was $200 \mathrm{IU}$. GnRHant injections were started on day 5 of stimulation. Similar to the ENGAGE trial, coasting was allowed up to 3 days, but hCG injection had to be withheld for women who had $>30$ follicles of $\geq 11 \mathrm{~mm}$ in any case. Up to two embryos were transferred 3-5 days after fertilization. 


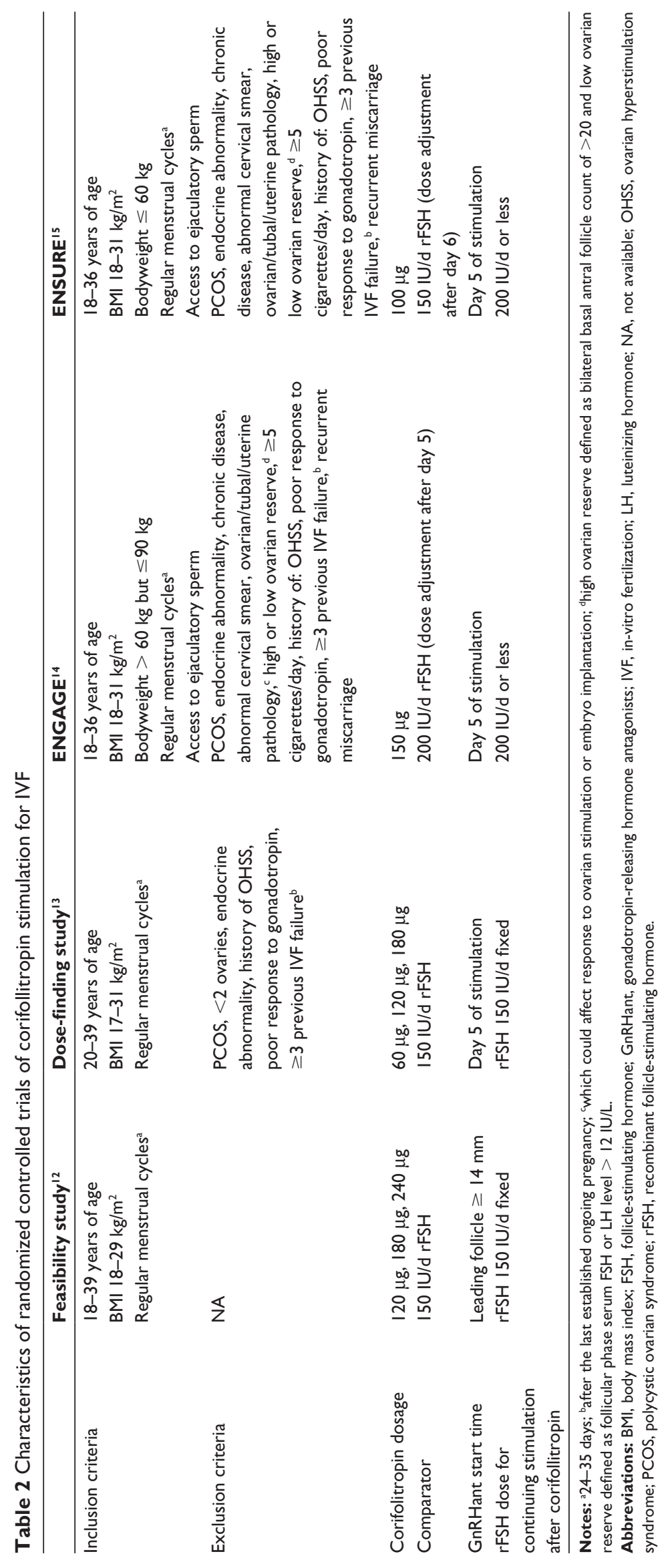




\section{The TRUST trial ${ }^{41}$}

The TRUST trial was a prospective multicenter, openlabel, uncontrolled clinical trial. The inclusion criteria and stimulation protocol were similar to the ENGAGE trial. ${ }^{14} \mathrm{~A}$ total of 682 women $>60 \mathrm{~kg}$ were recruited and underwent between one and three stimulated ART cycles and no more than six frozen thawed embryo transfer cycles from the first two treatment cycles. The number of women starting the first, second, and third COS cycles were 682, 375, and 198, respectively. Corifolitropin dose was $150 \mu \mathrm{g}$, and stimulation was continued with maximum of $225 \mathrm{IU} /$ day $\mathrm{rFSH}$ from day 8 onwards. GnRHant was started on stimulation day 5 or 6 . A maximum of three embryos were transferred 3-5 days after fertilization in fresh cycles. The results of the TRUST trials will be mentioned separately at the end of the next section.

\section{Outcomes}

Figures for embryology and clinical outcomes from the four RCTs are presented in Table 3.

\section{Follicular growth and oocyte yield}

The median duration of stimulation was similar at between 9 and 11 days in all groups of the four trials. ${ }^{12-15}$ The FS and the DFS reported detailed analysis of follicles of $\geq 11 \mathrm{~mm}$, $\geq 15 \mathrm{~mm}$, and $\geq 17 \mathrm{~mm}$ during the stimulation period. These trials had a focus on comparisons between different doses of corifollitropin alfa.

In the FS, the number of follicles $\geq 11 \mathrm{~mm}$ did not significantly differ between study groups throughout the stimulation period. Although the number of follicles in each size category tended to increase with increasing corifollitropin alfa dose, the dose-response relationship was only significant for follicles $\geq 15 \mathrm{~mm}$ on the day of hCG. In the overall analysis including all women who received hCG, regardless of the day of administration, the numbers of follicles in each category was similar across all study groups. However, in a subgroup analysis including women who already met the hCG criterion on day 8 , women in the $120 \mu \mathrm{g}$ group had significantly less follicles of $\geq 15 \mathrm{~mm}$ and $\geq 17 \mathrm{~mm}$, and women in the $180 \mu \mathrm{g}$ group had significantly less follicles of $\geq 17 \mathrm{~mm}$ compared with the rFSH group.

One woman in each corifollitropin alfa group (overall $3 / 74,4 \%)$ and one in the rFSH group $(1 / 25,4 \%)$ had cycle cancellation due to poor ovarian response either before or after hCG administration. One woman in the $120 \mu \mathrm{g}$ group and one in the $240 \mu \mathrm{g}$ group had cycle cancellation due to over response. The number of oocytes collected was similar for corifollitropin alfa groups and tended to be higher than

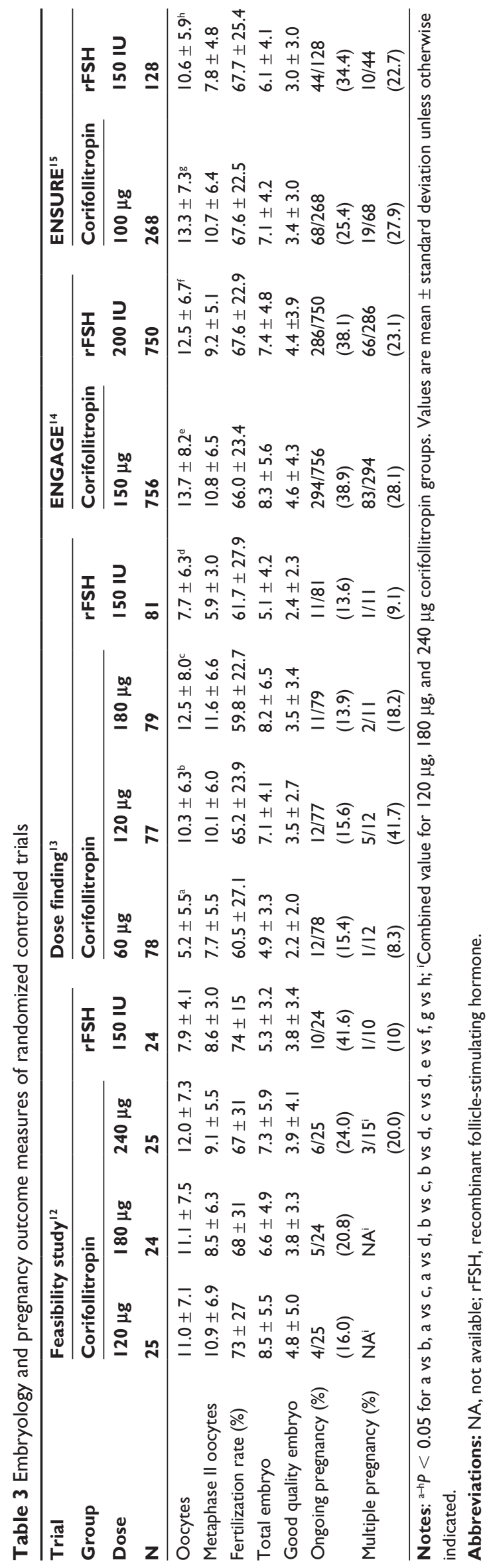


women given daily rFSH injections. The failure to observe a dose-response relationship across corifollitropin alfa groups was attributed to the high dose range used in the trial.

While the number and size of the follicles were similar at the initial assessment between the arms of the DFS, the number of follicles $\geq 11 \mathrm{~mm}$ was less in the $60 \mu \mathrm{g}$ group than in the 120 and $180 \mu \mathrm{g}$ groups from day 6 on. ${ }^{13}$ Accordingly, a high proportion of participants in the $60 \mu \mathrm{g}$ group $(22 / 78$, $28.2 \%$ ) were not given $\mathrm{hCG}$ and had their cycle cancelled due to insufficient ovarian response. The same figures were $2 / 77(2.6 \%), 3 / 79(3.8 \%)$, and $5 / 81(6.2 \%)$ in the $120 \mu \mathrm{g}$, $180 \mu \mathrm{g}$, and $\mathrm{rFSH}$ groups respectively. While all women who received hCG injection proceeded to fertilization in the 120 and $180 \mu \mathrm{g}$ groups, five women in the $60 \mu \mathrm{g}$ group and two women in the rFSH group had their cycle cancelled due to having too few follicles/oocytes after the hCG injection. Overall number of women having cycle cancellation due to low ovarian response summed up to $27 / 78(34.6 \%)$ in the $60 \mu \mathrm{g}$ group. On the other hand, two women $(2.6 \%)$ in the $120 \mu \mathrm{g}$ and one woman (1.3\%) in the $180 \mu \mathrm{g}$ group had cycle cancellation before hCG administration due to risk of hyperstimulation. No woman in the $60 \mu \mathrm{g}$ corifollitropin alfa or daily rFSH groups were considered at risk of hyperstimulation. The dose-response relationship between follicle counts and corifollitropin alfa dose reached significance by day 8 and was still apparent on the day of hCG injection. Compared with the rFSH group, the number of follicles in each size category tended to be higher in the 120 and $180 \mu \mathrm{g}$ corifollitropin alfa groups.

The order of the study groups according to number of cumulus corona complexes (COC) collected per started cycle was $60 \mu \mathrm{g}<\mathrm{rFSH}<120 \mu \mathrm{g}<180 \mu \mathrm{g}$, with statistically significant differences between categories. Trend analysis demonstrated a significant dose-response relationship between corifollitropin alfa dose and the number of COCs retrieved $(P<0.0001)$.

Based on the results from the two studies, it was decided that the appropriate dose for a single injection of corifollitropin alfa would be higher than $60 \mu \mathrm{g}$ and less than $240 \mu \mathrm{g}$ in women with an anticipated normal ovarian response. A predictive pharmacokinetic and pharmacodynamic modeling study was conducted using data from 2667 women who received corifollitropin alfa before, and it was suggested that the optimal dose, defined as the lowest dose providing the minimal cancellation rate, would be $100 \mu \mathrm{g}$ for women $\leq 60 \mathrm{~kg}$ and $150 \mu \mathrm{g}$ for women weighing $>60 \mathrm{~kg}{ }^{42}$

Using the doses suggested by the modeling study, women given $150 \mu \mathrm{g}$ corifollitropin alfa in the ENGAGE trial and women given $100 \mu \mathrm{g}$ corifollitropin alfa in the ENSURE trial had significantly more follicles of $\geq 11 \mathrm{~mm}$ on $\mathrm{hCG}$ day than their counterparts who started stimulation with 200 and 150 IU/day rFSH injections in respective trials. ${ }^{14,15}$ While dose decreases before day 7 were more commonly attempted in the corifollitropin alfa groups than in the daily FSH group in both trials $(11.3 \%$ versus $8.4 \%$ in the ENGAGE trial, and $6.4 \%$ versus $0.8 \%$ in the ENSURE trial), the proportion of women undergoing coasting for $\geq 2$ days were similar between the two arms of the ENGAGE trial (1.6\% versus $2.2 \%$ ). Women in the corifollitropin alfa groups had significantly more COCs collected per started cycle than women who started stimulation with daily rFSH injections in both trials. ${ }^{14,15}$ Estimated differences were +1.2 and $+2.7 \mathrm{COCs}$ in the ENGAGE and ENSURE trials, respectively $(P<0.001$ for both comparisons). Notably, one-third of women in the corifollitropin alfa arms (32.9\% and 32.8\% in the ENGAGE and the ENSURE trials, respectively) reached the hCG criterion before or on day 8 and did not require additional FSH injections. Pregnancy rate was $44 \%$ for such women in the ENGAGE trial.

\section{Hormonal profiles}

Serum FSH immunoreactivity increased rapidly until stimulation day 3 (post injection day 2) in recipients of corifollitropin alfa. The peak level depended on the corifollitropin alfa dose administered and exceeded the peak levels achieved in recipients of daily $\mathrm{rFSH}$ in both ENGAGE and ENSURE trials. There were no significant differences between serum FSH levels in corifollitropin alfa and $\mathrm{rFSH}$ groups from day 8 onward. ${ }^{14,15}$

Serum E2 levels tended to increase faster following corifollitropin alfa injection than after daily $\mathrm{rFSH}$ injections. ${ }^{12-15}$ This is consistent with the higher serum FSH levels and higher number of follicles $\geq 11 \mathrm{~mm}$ observed in the corifollitropin alfa arms during the initial days of stimulation. On stimulation day 8, serum E2 levels had a dose-response relationship with corifollitropin alfa dose..$^{13}$ However serum E2 levels on the day of hCG injection was not significantly different between corifollitropin alfa and rFSH groups. ${ }^{12-15}$

Inhibin B levels followed a similar pattern with serum E2 levels in all trials, and consistently increased during the first 6 days of stimulation. Similar to E2 levels, inhibin B levels were similar between corifollitropin alfa and daily $\mathrm{rFSH}$ regimens. The only exception was the sharp decline after day 6 in the $60 \mu \mathrm{g}$ corifollitropin alfa arm of the DFS. ${ }^{13}$ This observation is consistent with a lower number of growing 
follicles in recipients of the $60 \mu \mathrm{g}$ dose and further confirms that this dose is inadequate for COS.

LH levels declined rapidly after corifollitropin alfa injection in all groups. However, women who received the $240 \mu \mathrm{g}$ dose had increasing LH levels from the third day after corifollitropin alfa injection. ${ }^{12}$ Similar to women receiving lower corifollitropin doses, LH levels declined following commencement of GnRHant injections in these women. With the flexible GnRHant protocol used in the FS, LH rises before the start of the GnRHant injections were observed in 12 of 74 women $(16.2 \%)$ who received corifollitropin alfa and in 2 of 24 women $(8.3 \%)$ who had daily rFSH injections. ${ }^{12}$ Although the incidence of premature LH surges before starting GnRHant was not significantly different between the groups, this has led to starting GnRHant injections on the fifth day of stimulation in the following trials. Despite the early start of GnRHant, a premature LH rise of $\geq 10 \mathrm{IU} / \mathrm{L}$ was detected by day 5 in 12 of the 234 (5.1\%) women in the corifollitropin alfa arms of the DFS. ${ }^{13}$ None of the women in the $\mathrm{rFSH}$ arm had a premature LH rise before GnRHant injection in the same study. Notably, three women in the corifollitropin alfa arms and two in the rhFSH arm had a premature LH rise after GnRHant injections. ${ }^{13}$

The incidence of premature LH rises were $7 \%$ versus $2.1 \%(P<0.01)$ and $5.2 \%$ versus $3.9 \%(P=0.57)$ in the corifollitropin alfa and rFSH arms of the ENGAGE and ENSURE trials, respectively. ${ }^{14,15}$ The pregnancy rates for women with premature LH rises were not significantly different between corifollitropin alfa (45.3\%) and $\mathrm{rFSH}(31.3 \%)$ groups of the ENGAGE trial. ${ }^{14}$

The higher incidence of premature LH rises observed in the corifollitropin alfa groups seems to result from the higher FSH exposure during the early follicular phase. Similar observation has been reported when women with high ovarian reserve were given higher starting dose of daily rFSH injections in earlier trials evaluating GnRHant. ${ }^{38,43,44}$ The ENGAGE investigators regarded any impact of the higher incidence of LH rises in the corifollitropin alfa group negligible based on pregnancy rates achieved in women with a premature LH rise being similar to overall pregnancy rates achieved in the aforementioned early GnRHant trials. $^{43,44}$

Progesterone levels remained low throughout the stimulation period in all corifollitropin alfa groups and were not significantly different than women who received daily $\mathrm{FSH}$ injections. Likewise, luteal phase hormone profiles were not significantly different between corifollitropin alfa and $\mathrm{rFSH}$ groups. ${ }^{12,14,15}$

\section{Embryology laboratory parameters}

Fertilization rate was similar across different doses of corifollitropin alfa, as well as between corifollitropin alfa and $\mathrm{rFSH}$ groups in all trials. ${ }^{12-15}$

Although the number of embryos available tended to be higher in corifollitropin alfa arms (especially in the $120 \mu \mathrm{g}$ arm) of the FS, the numbers of good quality embryos were similar across all groups including the rFSH group. ${ }^{12}$ Women in the 120 and $180 \mu \mathrm{g}$ arms of the DFS had significantly higher numbers of total embryos and good quality embryos than women in the rFSH arm. ${ }^{13}$ These findings are in accordance with the trend towards higher numbers of COCs retrieved in these groups. The numbers of embryos available and good quality embryos were similar in corifollitropin alfa and $\mathrm{rFSH}$ arms of the ENGAGE and the ENSURE trials. ${ }^{14,15}$ The mean number (standard deviation) of embryos cryopreserved in the corifollitropin alfa and the $\mathrm{rFSH}$ groups were 4.3 (3.6) versus 3.9 (2.7) and 2.0 (3.0) versus 1.7 (2.6) in the ENGAGE and the ENSURE trials respectively. ${ }^{14,15}$

\section{Clinical outcome}

Despite the lack of a statistically significant difference between ongoing pregnancy rates between the four arms of the FS $(P=0.19)$, it should be noted that when all women in the corifollitropin alfa groups were compared with women in the $\mathrm{rFSH}$ group, the difference in ongoing pregnancy rates was significant $(15 / 74,20.3 \%$ versus $10 / 24,41.6 \%$; $P=0.04) .{ }^{12}$ However, it should also be noted that the high ongoing pregnancy rate achieved in the $\mathrm{rFSH}$ arm, despite lower number of oocytes retrieved, is extraordinary and could be a chance finding.

Incidence of vital pregnancy (defined as pregnancy with at least one fetus with a heart beat $>12$ gestational weeks) and ongoing pregnancy rates per cycle and per transfer was similar in the four arms of the DFS. ${ }^{13}$ Ongoing pregnancy rate per fresh cycle ranged between $16 \%$ and $24 \%$ in the corifollitropin alfa arms of the FS and the DFS. ${ }^{12,13}$ Cumulative ongoing pregnancy rate including transfer of frozen thawed embryos obtained from the index cycle within a year was only reported in the DFS, and figures were $18 \%$ in the $60 \mu \mathrm{g}, 27 \%$ in the $120 \mu \mathrm{g}, 24 \%$ in the $240 \mu \mathrm{g}$ corifollitropin alfa groups, and $20 \%$ in the daily rFSH group. ${ }^{13}$

Overall, higher ongoing pregnancy rates were achieved in the ENGAGE trial, which used the corifollitropin alfa dose adjusted for bodyweight and a higher starting dose of daily rFSH injections. ${ }^{14}$ Ongoing pregnancy rate per cycle was similar at $38.9 \%$ and $38.1 \%$ in the corifollitropin alfa and $\mathrm{rFSH}$ groups, respectively $(P=0.7) .{ }^{14}$ Overall, ongoing 
pregnancy rates achieved in the ENSURE trial, also using the bodyweight-adjusted corifollitropin alfa dose, seemed to be lower than the figures achieved in the ENGAGE trial. Ongoing pregnancy rate per cycle was $25.4 \%$ and $34.4 \%$ in the corifollitropin alfa and $\mathrm{rFSH}$ groups, respectively. Although the difference was not statistically significant at the 0.05 level $(P=0.06)$, the relative difference of almost $50 \%$ between the groups and borderline statistical significance are noteworthy. Given the similar stimulation characteristics in the two groups, ENSURE investigators consider the observed difference a chance finding. ${ }^{15}$ When the results from the two RCTs using bodyweight-adjusted gonadotropin dosages are combined, ongoing pregnancy rates were not significantly different between corifollitropin and daily rFSH groups (odds ratio $0.95,95 \%$ confidence interval $0.79-1.15$ ) (Figure 1).

\section{Outcomes of repeated corifollitropin COS cycles}

Total duration of stimulation, the numbers of COCs retrieved, numbers of embryos generated, good quality embryos, and embryos transferred were similar in the first, second, and third COS cycles of the TRUST trial. ${ }^{41}$ Embryo implantation, vital pregnancy and ongoing pregnancy rates seem consistent across fresh embryo transfer cycles. Cumulative ongoing pregnancy rate including spontaneous pregnancies and pregnancies achieved with frozen thawed embryo transfer between stimulated cycles was $61 \%$ after censoring for women who discontinued treatment. ${ }^{41}$ Efficacy results of the TRUST trial are presented in Table 4.

\section{Safety}

\section{Immunogenicity}

Corifollitropin alfa is a "designer" molecule with deviated carbohydrate site chains that are foreign to humans. In theory, corifollitropin alfa can be immunogenic and lead to drug-related hypersensivity. It is with this concern that the first study in humans was performed in men with hypogo- nadotropic hypogonadism to avoid future complications possibly raised by formation of cross-reactive antibodies against native $\mathrm{FSH}$ or $\mathrm{hCG} .{ }^{32}$ No anti-corifollitropin alfa or anti-CHO antibodies were detected in the participants of this Phase I study. This finding was confirmed in over 1000 women who received corifollitropin in other Phase II and III studies conducted to date..$^{11-13,15,37}$

The TRUST trial assessed immunogenicity and safety profile of corifollitropin in detail. ${ }^{41}$ Pre- and post-treatment serum samples were obtained and tested for the potential anticorifollitropin antibodies by a validated highly sensitive assay. Among the post-treatment samples obtained after 682 first, 375 second, and 192 third COS cycles, there was only one obtained after a second cycle that demonstrated statistically significantly increased binding affinity that was depletable by corifollitropin and rFSH, but not by recombinant LH or hCG. Isotyoping was technically impossible due to low titers in the sample. The sample actually lacked neutralizing activity and did not interfere with corifollitropin or rFSH bioactivity. The woman who provided this sample did not experience any adverse events (AEs), and another sample taken 6 months after treatment tested negative. She later conceived in a subsequent cycle with daily rFSH injections.

Participants of the TRUST study were systematically examined 30 minutes after each corifollitropin injection for injection site pain, itching, swelling, and redness. No drugrelated hypersensitivity reactions were reported. ${ }^{41}$ None of the participants had moderate or severe injection site reactions. These findings are essentially the same as reported in the former trials. ${ }^{13-15}$

\section{AEs}

A total of $46.8 \%, 35.2 \%$, and $31.3 \%$ of TRUST study participants reported at least one AE after cycles 1, 2, and 3 , respectively. ${ }^{41}$ Pain associated with oocyte collection procedure, pelvic pain, pelvic discomfort, headache, and

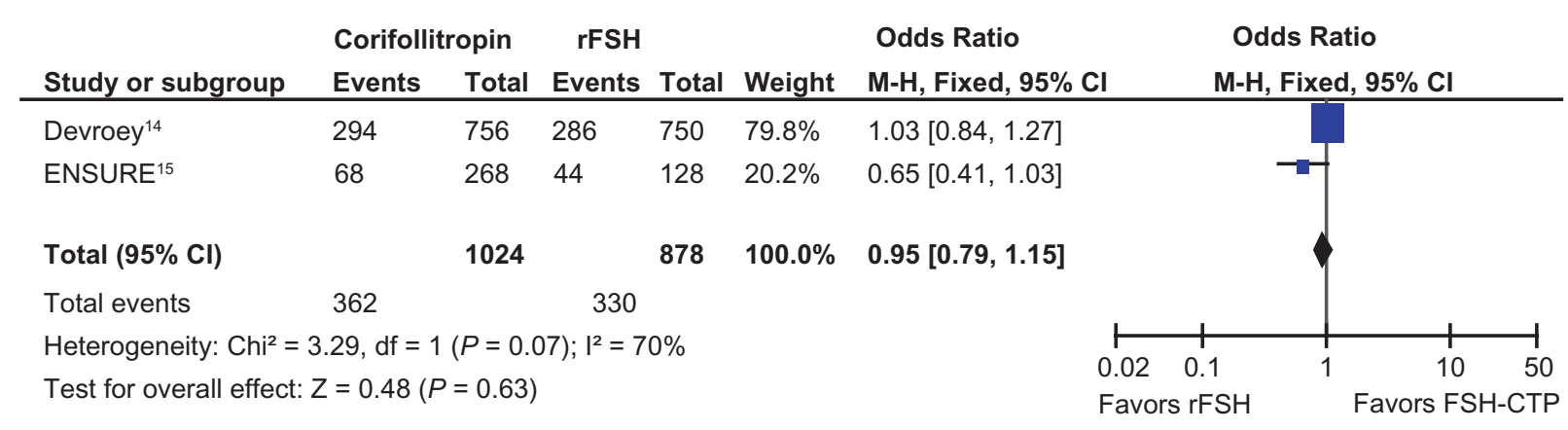

Figure I Ongoing pregnancy rates in trials using corifollitropin dose adjusted for bodyweight.

Abbreviations: $\mathrm{Cl}$, confidence interval; FSH-CTP, corifollitropin alfa; C-terminal peptide; $\mathrm{M}-\mathrm{H}$, Mantel-Haenszel; rFSH, recombinant follicle-stimulating hormone. 
Table 4 Efficacy results of the TRUST trial ${ }^{41}$

\begin{tabular}{llll}
\hline & $\begin{array}{l}\text { Cycle I } \\
(\mathbf{n}=\mathbf{6 8 2})\end{array}$ & $\begin{array}{l}\text { Cycle 2 } \\
(\mathbf{n}=\mathbf{3 7 5})\end{array}$ & $\begin{array}{l}\text { Cycle 3 } \\
(\mathbf{n}=198)\end{array}$ \\
\hline $\begin{array}{l}\text { Duration of stimulation } \\
\text { Number of COCs }\end{array}$ & 10 & $\mathrm{II}$ & 8 \\
$\begin{array}{l}\text { Number of embryos } \\
\text { generated }\end{array}$ & $6.4 \pm 4.9 \pm 7.2$ & $\begin{array}{l}11.5 \pm 6.8 \\
6.5 \pm 4.4\end{array}$ & $\begin{array}{l}11.3 \pm 7.6 \\
6.6 \pm 4.8\end{array}$ \\
$\begin{array}{l}\text { Number of good quality } \\
\text { embryos on day 3 }\end{array}$ & $3.2 \pm 3.1$ & $2.9 \pm 2.8$ & $2.8 \pm 2.7$ \\
$\begin{array}{l}\text { Number of embryos } \\
\text { transferred }\end{array}$ & $1.9 \pm 0.7$ & $2.1 \pm 0.7$ & $2.2 \pm 0.7$ \\
$\begin{array}{l}\text { Embryo implantation rate } \\
\text { Ongoing pregnancy rate }\end{array}$ & $21.2 \%$ & $16.6 \%$ & $12.2 \%$ \\
\hline Notes: & $22.7 \%$ & $20.5 \%$ & $20.7 \%$ \\
\hline
\end{tabular}

Notes: alncludes women with fresh embryo transfer; bregnancy with a heart beat $>10$ weeks. Values are mean \pm standard deviation unless otherwise indicated.

Abbreviation: COC, cumulus corona complex.

antepartum bleeding, together with mild injection site reactions, formed the majority of AEs. The incidence of these AEs in the TRUST study was similar to that in earlier reports. ${ }^{13-15}$ Severe AEs were uncommon, with incidence of $2.5 \%, 1.3 \%$, and $0.5 \%$ in cycles 1,2 , and 3 , respectively. ${ }^{41}$

A total of 63 serious AEs (SAEs) occurred in 47 participants (6.9\%) of the TRUST study. ${ }^{41}$ SAEs included eight ectopic pregnancies, two ruptured ectopic pregnancies, one heterotopic pregnancy, three missed abortions, and two imminent abortions. Only 15 SAEs were attributed to the study medication, including 10 occurrences of ovarian hyperstimulation syndrome (OHSS). ${ }^{41}$ Similarly six SAEs were reported in 5 of the $75(6.7 \%)$ and 20 of the 268 (7.5\%) women who received corifollitropin in the FS and the ENSURE trials, respectively. ${ }^{12,15}$ Proportion of women with SAEs were similar between the corifollitropin and $\mathrm{rFSH}$ arms in both the ENGAGE and ENSURE trials. Likewise, the nature of SAEs was similar in all studies.

\section{Ovarian hyperstimulation syndrome}

Sustained FSH levels and the inability for dose adjustment during the first week after corifollitropin injection raised concerns about OHSS risk. Increased follicular recruitment with corifollitropin injection as reflected by rapidly increasing serum E2 levels and the higher number of $>11 \mathrm{~mm}$ follicles observed within the first 6 days can justify such concern. Despite the fact that OHSS occurred with similar incidence in the corifollitropin and $\mathrm{rFSH}$ arms of individual trials $(5.4 \%$ versus $8.0 \%$ in the $\mathrm{FS}, 2.6 \%$ versus $2.4 \%$ in the DFS, $7.0 \%$ versus $6.3 \%$ in ENGAGE, and $6.7 \%$ versus $4.7 \%$ in ENSURE), it should be noted that women with PCOS and women who had a history of OHSS had been excluded from all trials. ${ }^{12-15,41}$ Similarly, women who had an over response or OHSS in a cycle were discontinued from the TRUST study. ${ }^{41}$ Therefore, these observations cannot be extrapolated to women with the highest risk of OHSS.

\section{Multiple pregnancy}

Except monozygotic twinning, multiple pregnancy rate following ART is determined by the number of embryos transferred. Number of embryos transferred were similar in corifollitropin and daily rFSH arms of the reviewed trials. As expected, this seems to result in similar multiple pregnancy rates. Multiple pregnancy rates are presented in Table 3.

\section{Conclusion and future research}

Corifollitropin alfa has a slower absorption rate and two-fold longer plasma $t_{1 / 2}$ than $r F S H$, while maintaining the same pharmocodynamic activity. This allows a single injection of corifollitropin alfa to effectively replace the first seven daily injections of rFSH in COS cycles for anticipated normoresponder women.

The optimal corifollitropin dose has been calculated to be $100 \mu \mathrm{g}$ for women with a body weight $\leq 60 \mathrm{~kg}$ and $150 \mu \mathrm{g}$ for women with a body weight $>60 \mathrm{~kg}$, respectively. Combination of corifollitropin with a fixed GnRHant protocol starting on stimulation day 5 seems to yield at least similar numbers of oocytes and good quality embryos, as well as similar ongoing pregnancy rates compared with women stimulated with daily rFSH injections.

The corifollitropin molecule does not seem to be immunogenic. Corifollitropin seems to have a favorable safety profile similar to daily rFSH injections. AE, SAE, and OHSS rates seem to be similar. Two points should be underlined regarding safety: firstly women with the highest risk of OHSS have been excluded from the trials and it remains to be seen how corifollitropin will perform in women with PCOS or high ovarian reserve. Secondly, as yet, there are no human data regarding congenital anomaly rate following corifollitropin stimulation available in the literature.

Further research is needed to determine whether corifollitropin can be used for women with anticipated poor or hyper-response. In theory, the increased follicular recruitment rate with corifollitropin can be expected to benefit poor responders.

It also remains to be objectively demonstrated whether switching to corifollitropin decreases anxiety and distress associated with ART. 


\section{Disclosure}

The authors report no conflicts of interest in this work.

\section{References}

1. Collins J. An international survey of the health economics of IVF and ICSI. Hum Reprod Update. 2002;8(3):265-277.

2. Mosher W, Pratt W. Fecundity and infertility in the United States, 1965-1988. Advance Data from Vital and Health Statistics, No. 192. Hyattsville, MD: National Center for Health Statistics; 1990.

3. Hughes EG, Fedorkow DM, Daya S, Sagle MA, Van de Koppel P, Collins JA. The routine use of gonadotropin-releasing hormone agonists prior to in vitro fertilization and gamete intrafallopian transfer: a meta-analysis of randomized controlled trials. Fertil Steril. 1992;58(5):888-896.

4. Tan SL, Maconochie N, Doyle P, et al. Cumulative conception and livebirth rates after in vitro fertilization with and without the use of long, short, and ultrashort regimens of the gonadotropin-releasing hormone agonist buserelin. Am J Obstet Gynecol. 1994;171(2):513-520.

5. Tan SL, Royston P, Campbell S, et al. Cumulative conception and livebirth rates after in-vitro fertilisation. Lancet. 1992;339(8806):1390-1394.

6. Domar AD, Zuttermeister PC, Friedman R. The psychological impact of infertility: a comparison with patients with other medical conditions. J Psychosom Obstet Gynaecol. 1993;(Suppl 14):45-52.

7. Olivius C, Friden B, Borg G, Bergh C. Why do couples discontinue in vitro fertilization treatment? A cohort study. Fertil Steril. 2004;81(2):258-261.

8. Verberg MF, Eijkemans MJ, Heijnen EM, et al. Why do couples dropout from IVF treatment? A prospective cohort study. Hum Reprod. 2008;23(9):2050-2055.

9. Al-Inany HG, Youssef MA, Aboulghar M, et al. Gonadotrophinreleasing hormone antagonists for assisted reproductive technology. Cochrane Database Syst Rev. 2011;(5):CD001750.

10. Griesinger G, Diedrich K, Tarlatzis BC, Kolibianakis EM. GnRHantagonists in ovarian stimulation for IVF in patients with poor response to gonadotrophins, polycystic ovary syndrome, and risk of ovarian hyperstimulation: a meta-analysis. Reprod Biomed Online. 2006;13(5):628-638.

11. Duijkers IJ, Klipping C, Boerrigter PJ, Machielsen CS, De Bie JJ, Voortman G. Single dose pharmacokinetics and effects on follicular growth and serum hormones of a long-acting recombinant $\mathrm{FSH}$ preparation (FSH-CTP) in healthy pituitary-suppressed females. Human Reprod. 2002;17(8):1987-1993.

12. Devroey P, Fauser BC, Platteau P, Beckers NG, Dhont M, Mannaerts BM. Induction of multiple follicular development by a single dose of long-acting recombinant follicle-stimulating hormone (FSH-CTP, corifollitropin alfa) for controlled ovarian stimulation before in vitro fertilization. J Clin Endocrinol Metabol. 2004;89(5):2062-2070.

13. The Corifollitropin Alfa Dose-finding Study Group. A randomized dose-response trial of a single injection of corifollitropin alfa to sustain multifollicular growth during controlled ovarian stimulation. Human Reprod. 2008;23(11):2484-2492.

14. Devroey P, Boostanfar R, Koper NP, Mannaerts BM, Ijzerman-Boon PC, Fauser BC. A double-blind, non-inferiority RCT comparing corifollitropin alfa and recombinant FSH during the first seven days of ovarian stimulation using a GnRH antagonist protocol. Human Reprod. 2009;24(12):3063-3072.

15. The Corifollitropin Alfa Ensure study group. Corifollitropin alfa for ovarian stimulation in IVF: a randomized trial in lower-body-weight women. Reprod Biomed Online. 2010;21(1):66-76.

16. Amin HK, Hunter WM. Human pituitary follicle-stimulating hormone: distribution, plasma clearance and urinary excretion as determined by radioimmunoassay. J Endocrinol. 1970;48(3):307-317.

17. Ryan RJ, Charlesworth MC, McCormick DJ, Milius RP, Keutmann HT. The glycoprotein hormones: recent studies of structure-function relationships. FASEB J. 1988;2(11):2661-2669.
18. Sowers JR, Pekary AE, Hershman JM, Kanter M, DiStefano JJ 3rd. Metabolism of exogenous human chorionic gonadotrophin in men. J Endocrinol. 1979;80(1):83-89.

19. Rizkallah T, Gurpide E, Vande Wiele RL. Metabolism of HCG in man. J Clin Endocrinol Metab. 1969;29(1):92-100.

20. Kohler PO, Ross GT, Odell WD. Metabolic clearance and production rates of human luteinizing hormone in pre- and postmenopausal women. J Clin Invest. 1968;47(1):38-47.

21. Kessler MJ, Mise T, Ghai RD, Bahl OP. Structure and location of the O-glycosidic carbohydrate units of human chorionic gonadotropin. J Biol Chem. 1979;254(16):7909-7914.

22. Matzuk MM, Hsueh AJ, Lapolt P, Tsafriri A, Keene JL, Boime I. The biological role of the carboxyl-terminal extension of human chorionic gonadotropin [corrected] beta-subunit. Endocrinology. 1990;126(1):376-383.

23. Creus S, Chaia Z, Pellizzari EH, Cigorraga SB, Ulloa-Aguirre A, Campo S. Human FSH isoforms: carbohydrate complexity as determinant of in-vitro bioactivity. Mol Cell Endocrinol. 2001;174(1-2):41-49.

24. D'Antonio M, Borrelli F, Datola A, et al. Biological characterization of recombinant human follicle stimulating hormone isoforms. Hum Reprod. 1999;14(5):1160-1167.

25. Miller C, Ulloa-Aguirre A, Hyland L, Chappel S. Pituitary follicle-stimulating hormone heterogeneity: assessment of biologic activities of each follicle-stimulating hormone form. Fertil Steril. 1983;40(2):242-247.

26. Fauser BC, Mannaerts BM, Devroey P, Leader A, Boime I, Baird DT. Advances in recombinant DNA technology: corifollitropin alfa, a hybrid molecule with sustained follicle-stimulating activity and reduced injection frequency. Human Reprod Update. 2009;15(3):309-321.

27. Weenen C, Pena JE, Pollak SV, et al. Long-acting follicle-stimulating hormone analogs containing N-linked glycosylation exhibited increased bioactivity compared with o-linked analogs in female rats. J Clin Endocrinol Metab. 2004;89(10):5204-5212.

28. Fares FA, Suganuma N, Nishimori K, LaPolt PS, Hsueh AJ, Boime I. Design of a long-acting follitropin agonist by fusing the $\mathrm{C}$-terminal sequence of the chorionic gonadotropin beta subunit to the follitropin beta subunit. Proc Natl Acad Sci US A. 1992;89(10):4304-4308.

29. LaPolt PS, Nishimori K, Fares FA, Perlas E, Boime I, Hsueh AJ. Enhanced stimulation of follicle maturation and ovulatory potential by long acting follicle-stimulating hormone agonists with extended carboxyl-terminal peptides. Endocrinology. 1992;131(6):2514-2520.

30. Verbost P, Sloot WN, Rose UM, de Leeuw R, Hanssen RG, Verheijden GF. Pharmacologic profiling of corifollitropin alfa, the first developed sustained follicle stimulant. Eur J Pharmacol. 2011;651(1-3):227-233.

31. Steelman SL, Pohley FM. Assay of the follicle stimulating hormone based on the augmentation with human chorionic gonadotropin. Endocrinology. 1953;53(6):604-616

32. Bouloux PM, Handelsman DJ, Jockenhovel F, et al. First human exposure to FSH-CTP in hypogonadotrophic hypogonadal males. Human Reprod. 2001;16(8):1592-1597.

33. Voortman G, van de Post J, Schoemaker RC, van Gerven JM. Bioequivalence of subcutaneous injections of recombinant human follicle stimulating hormone (Puregon ${ }^{\circledR}$ ) by Pen-injector and syringe. Hum Reprod. 1999;14(7):1698-1702.

34. Mannaerts BM, Rombout F, Out HJ, Coelingh Bennink H. Clinical profiling of recombinant follicle stimulating hormone (rFSH; Puregon): relationship between serum FSH and efficacy. Hum Reprod Update. 1996;2(2):153-161.

35. Voortman G, Mannaerts BM, Huisman JA. A dose proportionality study of subcutaneously and intramuscularly administered recombinant human follicle-stimulating hormone (Follistim*/Puregon) in healthy female volunteers. Fertil Steril. 2000;73(6):1187-1193.

36. le Cotonnec JY, Porchet HC, Beltrami V, Khan A, Toon S, Rowland M. Clinical pharmacology of recombinant human follicle-stimulating hormone. II. Single doses and steady state pharmacokinetics. Fertil Steril. 1994;61(4):679-686. 
37. Balen AH, Mulders AG, Fauser BC, et al. Pharmacodynamics of a single low dose of long-acting recombinant follicle-stimulating hormone (FSH-carboxy terminal peptide, corifollitropin alfa) in women with World Health Organization group II anovulatory infertility. J Clin Endocrinol Metab. 2004;89(12):6297-6304.

38. Fauser BC, Alper MM, Ledger W, Schoolcraft WB, Zandvliet A, Mannaerts BM. Pharmacokinetics and follicular dynamics of corifollitropin alfa versus recombinant FSH during ovarian stimulation for IVF. Reprod Biomed Online. 2010;21(5):593-601.

39. Devroey P, Fauser BC, Platteau P, Beckers NG, Dhont M, Mannaerts BM. Induction of multiple follicular development by a single dose of longacting recombinant follicle-stimulating hormone (FSH-CTP, corifollitropin alfa) for controlled ovarian stimulation before in vitro fertilization. J Clin Endocrinol Metab. 2004;89(5):2062-2070.

40. Beckers NG, Macklon NS, Devroey P, Platteau P, Boerrigter PJ, Fauser BC. First live birth after ovarian stimulation using a chimeric long-acting human recombinant follicle-stimulating hormone (FSH) agonist (recFSHCTP) for in vitro fertilization. Fertil Steril. 2003;79(3):621-623.
41. Norman RJ, Zegers-Hochschild F, Salle BS, et al. Repeated ovarian stimulation with corifollitropin alfa in patients in a $\mathrm{GnRH}$ antagonist protocol: no concern for immunogenicity. Hum Reprod. 2011;26(8): 2200-2208.

42. de Greef R, Zandvliet AS, de Haan AF, Ijzerman-Boon PC, Marintcheva-Petrova M, Mannaerts BM. Dose selection of corifollitropin alfa by modeling and simulation in controlled ovarian stimulation. Clin Pharmacol Ther. 2010;88(1):79-87.

43. Borm G, Mannaerts B. Treatment with the gonadotrophin-releasing hormone antagonist ganirelix in women undergoing ovarian stimulation with recombinant follicle stimulating hormone is effective, safe and convenient: results of a controlled, randomized, multicentre trial. The European Orgalutran Study Group. Hum Reprod. 2000;15(7): 1490-1498.

44. Fluker M, Grifo J, Leader A, et al. Efficacy and safety of ganirelix acetate versus leuprolide acetate in women undergoing controlled ovarian hyperstimulation. Fertil Steril. 2001;75(1):38-45.
International Journal of Women's Health

\section{Publish your work in this journal}

The International Journal of Women's Health is an international, peerreviewed open-access journal publishing original research, reports, reviews and commentaries on all aspects of women's healthcare including gynecology, obstetrics, and breast cancer. Subject areas include: Chronic conditions (migraine headaches, arthritis, osteoporosis);

\section{Dovepress}

Endocrine and autoimmune syndromes; Sexual and reproductive health; Psychological and psychosocial conditions. The manuscript management system is completely online and includes a very quick and fair peer-review system. Visit http://www.dovepress.com/ testimonials.php to read real quotes from published authors.

Submit your manuscript here: http://www.dovepress.com/international-journal-of-womens-health-journal 